\title{
Desarrollo y ambivalencias de la teoría económica de Marx*
}

\author{
Development and Ambivalences \\ of Marx's Economic Theory
}

\author{
Michael HEINRICH \\ Hochschule für Technik und Wirtschaft, Berlín
}

Recibido: 17/07/2010

Aceptado: 16/09/2010

\section{Resumen}

Con la profunda crisis económica a la que nos enfrentamos desde 2008 ha aumentado también el interés por la teoría marxiana en todo el mundo. Pero esto verdaderamente no es algo que deba sorprender: mientras que en la teoría económica de Marx las crisis desempeñan un papel importante, en la economía convencional son prácticamente ignoradas. En ésta aparecen como meros accidentes, que podrían ser evitados por medio de mercados flexibles (ésa es la creencia de los economistas neoclásicos) o por medio de una política económica adecuada (la creencia de los keynesianos).

Pero al hablar de la teoría económica marxiana deberíamos tener siempre en cuenta que hay un cierto desarrollo en el pensamiento de Marx, así como hay ciertas ambivalencias en conceptos teóricos básicos. En el marxismo tradicional, que en su mayoría ha intentado presentar la teoría marxiana como un edificio completo y perfecto, esas ambivalencias eran a menudo obviadas o incluso negadas. A continuación voy a dar una breve descripción de las principales etapas del desarrollo teórico de Marx, y después me centraré en las ambivalencias de su obra teórica de madurez.

\footnotetext{
* Texto de la ponencia presentada en inglés en el Congreso "Pensar con Marx. Potencia de una obra y actualidad de un proyecto", Facultad de Filosofía de la Universidad Complutense de Madrid, 24 de febrero de 2010. Traducción de César Ruiz Sanjuán.
} 


\begin{abstract}
With the deep economic crisis we face since 2008 also the interest in Marxian theories increased worldwide. This was not really surprising: while in Marx's economic theory crises play an important role, they are nearly neglected in mainstream economics. They appear there as mere accidents, which could be prohibited by flexible markets (this is the belief of neoclassical economists) or by an appropriate economic policy (the belief of the Keynesians).

But talking about Marxian economic theory, we should always have in mind, that there is a certain development in Marx's thinking as well as there are certain ambivalences in basic theoretical concepts. In traditional Marxism, which mostly tried to present Marxian theory as a complete and perfect building, especially such ambivalences were often negIected or even denied. In the following I will give a short overview about the main stages of Marx's theoretical development, and then I will focus on the ambivalences of Marx's mature theoretical work.
\end{abstract}

\title{
1. Principales etapas del desarrollo teórico de Marx
}

a) 1844: "Manuscritos económico-filosóficos", que estaban centrados en una concepción de la esencia del ser humano (Wesen des Menschen) y la alienación (Entfremdung) en el capitalismo, fuertemente influenciados por la filosofía de Feuerbach. En este momento, Marx tenía solamente unos pocos conocimientos tanto de teoría económica como de la evolución histórica del capitalismo.

b) 1845: Crítica de la filosofía feuerbachiana en las "Tesis sobre Feuerbach" y la "Ideología alemana", lo que fue al mismo tiempo una autocrítica a sus escritos de 1844. De ahora en adelante, afirmó Marx en la "Ideología alemana", la investigación empírica debe sustituir a la especulación filosófica. Pero en este momento, eso era sólo un programa.

c) 1845-49: Marx aceptó la teoría económica de David Ricardo como una descripción adecuada del capitalismo (criticando simplemente que Ricardo consideraba el capitalismo como algo que es eterno). Sobre esta base Marx criticó en "Miseria de la filosofía" (1847) el socialismo de Proudhon. Marx también aceptó la teoría de las clases proporcionada por los historiadores franceses, utilizándola para el "Manifiesto comunista" (1848). En este periodo Marx hizo un uso crítico de la economía política burguesa, pero no hizo una crítica categorial de la economía política.

d) 1850-57: En 1849, Marx tuvo que emigrar a Londres, que en ese momento era el mejor lugar para observar el capitalismo, y la biblioteca del Museo británico el mejor lugar para estudiar la economía política. Marx comenzó con sus estudios 
económicos desde el principio (véase especialmente los "Cuadernos de Londres" de 1850-53). Ahora comenzará la crítica categorial de Ricardo, que se desarrollará hasta convertirse en una crítica que abarcará toda la economía política.

e) 1857-63: Marx diseñó y realizó parcialmente su proyecto global de una "crítica de la economía política" en seis libros: capital, propiedad de la tierra, trabajo asalariado, Estado, comercio exterior, mercado mundial. Escribió los "Grundrisse" (1857/58), el "Urtext" (1858), publicó la "Contribución a la crítica de la economía política" (1859) como la primera parte de la "Crítica" planeada y escribió en 186163 una continuación de más de 2.000 páginas que permaneció inédita (hoy en día denominada "Ökonomisches Manuskript 1861-63", que incluye las "Teorías sobre el plusvalor").

f) 1863 - 1867: Marx cambió su plan original en 1862/63. Fue diseñado " $E l$ Capital", que constaba de cuatro libros. Pero "El Capital" no sólo debía incluir el libro del capital del plan de seis libros, sino también las principales partes teóricas de los libros sobre propiedad de la tierra y trabajo asalariado1. En 1863-65 fue escrito el primer borrador de los tres libros "teóricos" (el cuarto libro debía abarcar la historia de la teoría económica), el primer libro fue publicado en 1867. Una segunda edición considerablemente reelaborada apareció en 1872/73. En la teoría del valor (especialmente por lo que se refiere a la forma del valor y al fetichismo de la mercancía) podemos ver un desarrollo considerable entre 1859 y 1872.

g) Entre 1868 y 1881 Marx escribió varios manuscritos para los libros segundo y tercero, pero no logró completar "El Capital". Sólo después de su muerte (1883), su amigo y camarada Friedrich Engels editó los libros segundo y tercero (no existía un manuscrito del libro cuatro, las famosas "Teorías sobre el plusvalor" no son un borrador de este libro). A fin de hacer estos libros más "legibles", Engels realizó un buen número de cambios y reordenó el material que encontró en los manuscritos de Marx. Sólo en nuestros días, después de que los manuscritos originales de Marx han sido publicados en MEGA (Marx Engels Gesamtausgabe) ${ }^{2}$, podemos analizar las diferencias (a veces considerables) entre los manuscritos de Marx y la edición de Engels (véase Heinrich 1996/97).

\footnotetext{
1 El hecho de que "El Capital" de cuatro volúmenes era realmente un nuevo proyecto y no simplemente el plan de seis libros abreviado lo muestro con cierto detalle en Heinrich (2009).

2 Marx Engels Gesamtausgabe (MEGA) es una edición histórico-crítica de todos los trabajos, documentos y cartas de Marx y Engels, que se publica desde 1975 en Berlín. Abarca cuatro secciones: la sección I comprende todas las obras de Marx y Engels excepto "El Capital”, la sección II contiene "El Capital" y los manuscritos preparatorios, la sección III incluye todas las cartas escritas por Marx y Engels, además de todas las cartas que recibieron, y la sección IV comprende todos los extractos y notas realizados por Marx y Engels. Todos los manuscritos se editan en su forma y en su lengua original, sin intervenciones editoriales. Hasta ahora se han publicado más de 60 volúmenes, la MEGA completa abarcará alrededor de 115 volúmenes. Cada volumen consta de dos libros, uno con los textos y el segundo con descripciones detalladas de los manuscritos, registros y comentarios.
} 


\section{Revolución científica: ruptura con el campo teórico de la economía política}

Marx no sólo construyó una nueva teoría económica, su proyecto de una "crítica de la economía política" estaba dirigido a la crítica de toda una ciencia ${ }^{3}$ - con una finalidad política: Marx escribió acerca de "El Capital" que es el más "terrible misil" que se ha arrojado jamás a la burguesía (véase su carta a Becker del 17 de abril de 1867).

Podemos hablar de una "revolución científica" cuando un nuevo enfoque incluye una ruptura con el campo teórico (lo que significa los supuestos básicos que se tienen por evidentes) que constituía el fundamento de diferentes teorías que operan en este campo. La "Crítica de la economía política" de Marx formulada a partir de 1857 conlleva una ruptura con un campo teórico que caracteriza no sólo a la economía política clásica del siglo XVIII y principios del XIX, sino también la teoría neoclásica de finales XIX y del siglo XX. Los elementos básicos con los que Marx rompió los resumo como antropologismo, individualismo, empirismo y ahistoricismo (para una discusión extensa, véase Heinrich 2006):

a) Antropologismo: La hipótesis de que existe una cierta "esencia del ser humano" (Wesen des Menschen) desde la cual podemos entender las acciones humanas. En 1844 Marx sostiene también este punto de vista. Pero desde 1845 criticó la asunción de tal "esencia" como "especulación" 4 . Hay algunos atributos muy generales de los seres humanos, pero no son suficientes para explicar la acción humana o el carácter de la sociedad humana.

b) Individualismo: La sociedad se concibe como una expresión de los individuos y sus acciones. La base para entender la sociedad no son las relaciones sociales, sino los individuos. En los "Grundrisse" Marx reconoció claramente que la sociedad no consta de individuos, sino de relaciones sociales que definen las posiciones para los individuos 5 .

c) Empirismo: Se considera evidente que la economía y la sociedad son perfectamente transparentes, que se puede entender todo con un cuidadoso protocolo de

\footnotetext{
${ }^{3}$ Marx habló de un "intento científico" de "revolucionar una ciencia", ver su carta a Kugelmann del 28 de diciembre de 1862.

4 Fue Louis Althusser en "Pour Marx" (1965) quien insistió en este punto con el fin de mostrar que hay una ruptura entre el Marx "joven" (ideológico) y el Marx "maduro" (científico). A pesar de que se trata de una contribución pionera a la discusión, debe destacarse que el desarrollo del "joven" Marx al "maduro" no puede reducirse a una ruptura simple, hay distintas dimensiones en las que tuvieron lugar rupturas en diferentes momentos. Por otra parte, no hay un único Marx maduro, también el Marx maduro mostró avances considerables. Y por último, la contraposición de Althusser entre ideología y ciencia es problemática.

5 Véase, por ejemplo, "la sociedad no consiste en individuos, sino que expresa la suma de relaciones y condiciones en las que estos individuos están recíprocamente situados" (Die Gesellschaft besteht nicht aus Individuen, sondern drückt die Summe der Beziehungen, Verhältnisse aus, worin diese Individuen zu einander stehen, MEGA II/1.1, p. 188).
} 
hechos empíricos. No hay nada que revelar, todo es visible. Marx aceptó este punto de vista a partir de 1845. Pero después de 1857 se hizo cada vez más consciente de que el fetichismo y las mistificaciones ocultan (estructuralmente, no como resultado de un acto intencional) las estructuras internas del capitalismo. Simplemente por medio de exposiciones empíricas no se pueden revelar estas estructuras internas.

d) Ahistoricismo: No se niega el desarrollo histórico, pero se restringe a momentos meramente superficiales. Las estructuras básicas de la economía son siempre las mismas: o "naturales" (economía de mercado) o artificiales, distorsionadas (economía de no-mercado). Marx distingue diferentes modos de producción. Reconoció que hay formaciones sociales históricamente diferentes, que no pueden reducirse a una estructura básica común.

\section{Ambivalencias en la teoría económica de Marx}

En cualquier texto complejo podemos encontrar ambivalencias, ambigüedades, puntos poco claros, que permiten diferentes interpretaciones. Pero existen distintos tipos de ambivalencias. Pueden ocurrir accidentalmente o como expresión de algunos problemas conceptuales. En "El Capital" las ambivalencias fundamentales que tengo en mente son resultado de dos fuentes diferentes. Una fuente es el intento de Marx de popularizar su exposición. En algunos puntos este intento perjudicó el desarrollo claro de las categorías.

Otra fuente puede encontrarse en el carácter específico de "El Capital" de Marx. Como he afirmado anteriormente, "El Capital", o en términos más generales, la "Crítica de de la economía política " de Marx es una revolución científica, una ruptura con el campo teórico de la economía política clásica. Pero esta revolución científica, esta ruptura no fue ejecutada completamente por Marx. En algunos puntos de su exposición, Marx permaneció en el campo con el que acababa de romper. En el mismo texto podemos observar una ruptura con este campo y la continua presencia de algunos elementos de este campo. Estos dos lados no están claramente separados. No podemos distinguirlos, por ejemplo, a lo largo de la línea de los capítulos. Estos dos lados constituyen dos discursos, que siempre están presentes, que interfieren entre sí de diferentes maneras, y que dan razón a distintas interpretaciones. La interferencia de estos dos discursos causa problemas específicos en la argumentación de Marx, como por ejemplo el conocido "problema de la transformación" (la transformación de los valores en los precios de producción). Examino extensamente estos dos discursos en mi libro "Die Wissenschaft vom Wert". Aquí voy a intentar aclarar mi tesis discutiendo brevemente algunas cuestiones básicas. 


\subsection{Teoría sustancialista-naturalista del valor o teoría monetaria del valor}

Las ambivalencias empiezan ya con el valor, la forma de valor y el dinero. Nos podemos imaginar lo difíciles que han sido estas cuestiones para Marx si recordamos no sólo que Marx mismo consideraba el capítulo sobre el valor y la mercancía como el más difícil de su libro (véase el prefacio de la primera edición de "El Capital"), sino también que cambió diversas veces la exposición del material de este capítulo. Marx escribió al menos siete variantes (de las cuales quedan seis) con diferencias notables en la argumentación. Las podemos encontrar en:

- "Grundrisse" (1857/58), si entráramos en detalles, ya aquí podríamos distinguir diferentes intentos de comprender la relación entre valor y dinero.

- "Urtext" (1858), aquí se han perdido las partes sobre el valor y la forma de valor, pero quedaron partes importantes que se ocupan del dinero.

- "Contribución a la crítica de la economía política" (1859).

- Primera edición del libro I de "El Capital”, capítulo 1 (1867).

- Apéndice a la primera edición del libro I de "El Capital" (1867).

- Un manuscrito escrito entre diciembre de 1871 y enero de 1872 que preparaba la segunda edición.

- Segunda edición del libro I de "El Capital” (1872/73).

En términos muy abreviados, mi tesis es que en "El Capital" podemos encontrar elementos de dos enfoques distintos del valor: un "enfoque sustancialista-naturalista del valor" y un "enfoque monetario del valor".

El enfoque sustancialista del valor refiere el valor a la mercancía individual y al trabajo que se necesita para producir esta mercancía individual. Desde este punto de vista la determinación social sólo tiene lugar porque el trabajo es únicamente generador de valor en tanto que es "tiempo de trabajo socialmente necesario". Pero el valor parece no tener nada que ver con otras mercancías, existe como un tipo de sustancia independiente dentro de la mercancía individual. Leídas superficialmente, se pueden entender de esta manera las primeras cuatro o cinco páginas de "El Capital". Este punto de vista sustancialista ha dominado en el marxismo tradicional. De hecho, a menudo sólo fueron tomadas en serio las primeras cuatro o cinco páginas del primer capítulo, y en general se descuidaron las siguientes cincuenta páginas.

Combinado con este punto de vista sustancialista del valor se presenta un punto de vista naturalista del trabajo abstracto. El trabajo abstracto, escribió Marx al final del párrafo 2 del capítulo uno, es trabajo "en el sentido fisiológico", gasto de cerebro, músculos, nervios y mano. Desde luego, cualquier trabajo es gasto de cerebro, nervios 
y músculos, pero la pregunta es: ¿es este atributo general de cualquier trabajo realmente lo determinante de una cualidad social como el trabajo abstracto?6.

Se puede mostrar que este enfoque sustancialistas y naturalista del valor y no abandona realmente el campo teórico de la economía política clásica. Este enfoque es una mejora de la economía clásica, una formulación más precisa, pero no una ruptura.

Sólo en el segundo enfoque existe una ruptura real con el campo teórico de la economía política. Una teoría no-sustancialista del valor muy clara y una determinación anti-naturalista del trabajo abstracto las podemos encontrar en algunas argumentaciones de la "Contribución a la crítica de economía política", en los subcapítulos sobre la forma de valor y el fetichismo de la mercancía en "El Capital", y especialmente en las reflexiones de Marx sobre su modo de exposición en el manuscrito que fue escrito en el invierno de 1871/72, cuando Marx realizó los cambios de la primera edición de "El Capital" a la segunda.

Especialmente este último manuscrito, que fue publicado por primera vez en 1987, es extremadamente interesante, porque incluye consideraciones que no podemos encontrar en "El Capital", ni en la primera ni en la segunda edición. En este manuscrito Marx aclara, entre otras cosas, que su propia exposición producía la apariencia de que se pudiera hablar de una mercancía individual y del valor de esta mercancía individual. Pero hablado estrictamente esto es erróneo: el valor y la mercancía sólo existen cuando hay una relación de al menos dos mercancías.

En este manuscrito se dice que si se cambian una chaqueta y una tela, se "reducen a la objetivación de trabajo humano como tal". Pero con esta reducción no debe olvidarse

"que ninguna es para sí tal objetividad de valor, sino que sólo lo son en tanto que es una objetividad común a ambas. Fuera de su mutua relación - la relación en la que se igualan - ni la chaqueta ni la tela poseen objetividad de valor, esto es, objetividad como gelatina de trabajo humano en cuanto tal" (Aber in dieser Reduktion wurde vergessen, daß keines für sich solche Werthgegenständlichkeit ist, sondern daß sie solches nur sind, soweit das ihnen gemeinsame Gegenständlichkeit ist. Ausserhalb ihrer Beziehung auf einander der Beziehung worin sie gleichgelten besitzen weder Rock noch Leinwand Werthgegenständlichkeit oder ihre Gegenständlichkeit als Gallerten menschlicher Arbeit schlechthin, MEGA II/6, p. 30).

Esto tiene como consecuencia que

"un producto del trabajo, considerado para sí aisladamente, no es valor, del mismo modo que no es mercancía. Sólo llega a ser valor en su unidad con otros productos del

6Ya el economista soviético Isaac Rubin, que fue ejecutado en 1937 durante la "Gran Purga", insistió sobre este punto en su notable libro "Ensayos sobre la teoría marxiana de valor" (Moscú, 1928). 
trabajo“ (Ein Arbeitsprodukt, für sich isolirt betrachtet, ist also nicht Werth, so wenig wie es Waare ist. Es wird nur Werth in seiner Einheit mit andrem Arbeitsprodukt, MEGA II/6, p. 31) ${ }^{7}$.

Esto tiene consecuencias importantes. El valor no sólo depende de una sustancia social, depende de una sustancia que no puede existir en una cosa individual y que no está determinada por la sola producción. El carácter específico de esta sustancia es la causa por la que Marx utilizó un buen número de metáforas cuando escribió sobre ello. Estas metáforas como "objetividad fantasmal" (gespenstige Gegenständlichkeit, MEW 23, p. 52) u "objetividad puramente fantástica" (rein phantastische Gegenständlichkeit, MEGA II/6, p. 32) son ignoradas con frecuencia por los lectores. Pero estas metáforas transmiten información importante acerca de esta muy específica sustancia no-sustancialista del "valor". (Esta noción no-sustancialista de sustancia es también una ruptura radical con más de 2000 años de uso filosófico de la noción de sustancia).

El análisis de la forma de valor (parte 3 del capítulo 1 de "El Capital") pone de manifiesto que el valor sólo puede existir cuando hay una expresión del valor autónoma y general. Y esta expresión general del valor, como se muestra en el capítulo 2 de "El Capital", es el dinero. Así que el dinero no es sólo una característica adicional, una herramienta técnica para hacer más fácil el intercambio (éste es el punto de vista de la economía clásica y neoclásica). El dinero es fundamental para la existencia de la objetividad de valor como una forma social general. Por consiguiente, podemos hablar de una teoría monetaria del valor 8.

Esta acentuación del dinero no niega la conexión del valor y el trabajo, pero hay que tener cuidado: es una conexión del valor y el trabajo abstracto. Y el trabajo abstracto, como Marx señaló en la "Contribución a la crítica de economía política", se basa en una "igualación objetiva de trabajos desiguales que efectúa por la fuerza el proceso social" (MEW 13, p. 45; p. 44)9. Por lo tanto, el trabajo abstracto es una abstracción forzada de las diferencias del trabajo, una abstracción que sólo está presente en el cambio. El hecho de que valor sólo esté presente en el cambio no significa que sea el cambio lo que crea el valor o lo que es la causa del valor. Aunque el valor sólo está presente en el cambio, el valor es un efecto de la producción y el cambio.

\footnotetext{
${ }^{7}$ Las partes decisivas de este manuscrito están publicadas y comentadas en Heinrich (2008), que aparecerá este año en traducción española.

8 En Alemania Occidental la discusión sobre el carácter monetario de la teoría marxiana del valor comenzó en la década de 1970 con los artículos de Hans Georg Backhaus, ahora recogidos en Backhaus (1997).

${ }^{9}$ Cuando existe traducción española de la obra, se indica la página de la misma a continuación de la página de la edición alemana (N. del T.).
} 
Como resultado de estos argumentos podemos ver que la relación entre valor y dinero es mucho más estrecha que en la economía política clásica y neoclásica, y también más estrecha de lo que la describe la corriente principal del marxismo tradicional: el valor no es sólo el fundamento del dinero, sino que el valor no puede existir sin el dinero. El enfoque sustancialista, que ha dominado en el marxismo tradicional, puede asumir la primera parte de esta frase, pero la segunda parte ni siquiera es comprensible para este enfoque.

\subsection{Determinaciones económicas formales y acción humana}

El análisis de la forma de valor (que podemos encontrar en la parte 3 del capítulo 1 de "El Capital") es una parte extremadamente importante de "El Capital". También aquí podemos encontrar un tipo especial de ambivalencia, que tiene sus raíces en el cambio a una "forma más popular" de exposición en la segunda edición de "El Capital" (un cambio que comienza ya con el apéndice de la primera edición). En el apéndice y en la segunda edición, Marx cambió la secuencia de las formas de valor. Introdujo la forma de dinero como la cuarta forma y abandonó la auto-contradictoria "Forma IV" del primer capítulo de la primera edición.

Este cambio veló la diferencia fundamental entre el análisis de la forma mismo (el contenido del capítulo 1) y el análisis de la acción orientado a la forma, que es el contenido del capítulo 2. Aquí, en el capítulo 2, aparecen por primera vez los propietarios de mercancias, mientras que en el capítulo 1 se han investigado sólo las determinaciones formales, no la acción de los propietarios de mercancías. El dinero es el resultado de la acción colectiva (no una acción deliberada, por supuesto) de los propietarios de mercancías. Por consiguiente, la transición de la forma general de valor a la forma de dinero en la segunda edición no está enraizada en un argumento analítico-formal, sino en un argumento que tiene que ver con la acción: Marx lo llamó la "costumbre social" (gesellschaftliche Gewohnheit, MEW 23, p. 84; p. 86).

Como consecuencia de este cambio, en muchas discusiones marxistas el estatus y el objetivo del capítulo 2 de "El Capital" permanecieron inaclarados. Si el dinero ya fue el resultado del análisis de la forma de valor del capítulo 1, ¿por qué un segundo capítulo que también presenta el dinero como resultado? Compruébese la literatura sobre "El Capital". Por lo general no se encontrará ninguna verdadera respuesta a esta pregunta. A veces se dice que el capítulo 2 es "más concreto", pero no se explica por qué habría de ser necesario un capítulo "más concreto".

La diferencia categorial decisiva es que el capítulo 1 presenta un análisis de la forma de mercancía (abstrayendo de los propietarios de mercancías) y que el capítulo 2 analiza las acciones necesarias de las personas en el marco de estas formas. 
En el mundo real las determinaciones formales y la acción humana existen siempre simultáneamente. Pero en la exposición científica el análisis de las formas sociales tiene prioridad sobre el análisis de la acción humana: el análisis de la forma es necesario para explicar la acción humana típica. Sólo a causa de esta prioridad, Marx pudo decir en el prefacio a la primera edición de "El Capital" que el capitalista y el terrateniente sólo se toman en consideración como "personificaciones de categorías económicas" (MEW 23, p. 16; p. 8). Y ésta es también la razón por la que Marx nunca realizó una crítica de los capitalistas, sino una crítica del capitalismo (una diferencia que es confundida a veces también en la izquierda).

\subsection{El problema de la mercancía dinero}

Pero hay otro problema conceptual en el análisis de la forma de valor que no tiene sus raíces en el cambio a una "forma más popular": la mercancía dinero.

En su análisis de la forma de valor, Marx presupone la necesidad de una mercancía dinero. En las relaciones de intercambio reales no tiene que estar presente la mercancía dinero, puede ser sustituida, como Marx ya observó en el capítulo 3 de "El Capital". Pero, según Marx, los signos circulantes sólo son sustitutos de la mercancía dinero. Ésta debería ser algo parecido a un ancla de todo el sistema monetario. Hablando en un plano teórico, Marx conceptualizó su teoría del dinero como si la existencia de una mercancía dinero fuera absolutamente necesaria para cualquier sistema monetario.

Si esta conceptualización fuera correcta, no podríamos entender el sistema monetario contemporáneo con las categorías marxianas, porque este sistema no depende de una mercancía dinero, ni legalmente (desde el fin del sistema de Bretton Woods), ni realmente (no hay ninguna mercancía dinero especial: que los bancos centrales posean oro es una reliquia histórica y desde hace años intentan deshacerse de él mediante la venta de pequeñas porciones, lo que no dará lugar a una caída del precio del oro).

Afortunadamente la conceptualización de Marx no es correcta. En su teoría del valor no hay una necesidad categorial de una mercancía dinero. Lo que Marx demostró en el análisis de la forma de valor fue la necesidad de la forma de dinero (estrictamente hablando: la necesidad de la forma general de equivalente). Pero no demostró que el portador de esta forma debe ser él mismo una mercancía. Marx solamente presupuso que este portador es una mercancía.

En el primer capítulo de la primera edición de "El Capital" podemos encontrar un importante indicio de por qué no es necesario que el portador de la forma de dinero tenga que ser una mercancía. En la primera edición, Marx dejó claro que el dinero es un universal muy especial: El dinero es un universal que existe como indi- 
viduo al mismo nivel que los individuos particulares. Marx utiliza una imagen muy plática para ilustrar esto: es como si además de los leones, tigres, aves, etc. concretos, también existiera "el animal" caminando entre ellos (MEGA II/5, p. 37).

Continuando con esta consideración, podemos concluir que "el animal" no puede existir realmente como un individuo junto a los leones, tigres concretos, etc. Tiene haber "algo" que es aceptado como "el animal", que es una representación de "el animal". "El animal" puede ser representado por un determinado animal, el león, por ejemplo. Entonces el león no sólo cuenta como león, sino también como "el animal". O "el animal" puede ser representado por una placa con la letra "A", por ejemplo.

En resumen: en el nivel de análisis de la forma de valor en el libro I de "El Capital" no es posible determinar el carácter del algo concreto que representa al universal como individuo. Este algo puede ser una mercancía o un puro signo. Una mercancía dinero puede existir, pero su existencia no es necesaria.

Al nivel de la teoría del crédito, que Marx comenzó a desarrollar en el libro III de "El Capital", es posible demostrar que un sistema monetario basado en una mercancía dinero sólo puede existir por un periodo limitado de transición histórica. Aunque Marx mismo no saca esta conclusión, su enfoque del sistema de crédito nos proporciona todos los elementos para esta demostración (véase Heinrich 2006, capítulo 6.3).

En el prefacio a la primera edición de "El Capital", Marx puso de manifiesto que no quería analizar sólo un período especial del desarrollo capitalista, sino el capitalismo como tal. Y al final del libro III estableció que su objetivo es presentar la "media ideal" de la "organización interna del modo de producción capitalista" (MEW 25, p. 839; p. 1057). Sin embargo, la mercancía dinero no pertenece a esta "media ideal". En este caso Marx confundió un atributo transitorio del sistema monetario capitalista con su "media ideal".

\subsection{La transición categorial ausente del dinero al capital}

De modo similar a la teoría monetaria del valor, hay también una teoría monetaria del capital. Esto es algo muy claro en los "Grundrisse" y, sobre todo, en el "Urtext" (MEGA II/2) donde podemos encontrar la así denominada "transición del dinero al capital", una transición categorial donde Marx argumenta que el dinero como una forma de valor independiente y duradera sólo puede existir cuando hay no sólo el movimiento $\mathrm{M}-\mathrm{D}-\mathrm{M}$, sino también el movimiento $\mathrm{D}-\mathrm{M}-\mathrm{D}$, que implica D - M - D'. Por lo tanto, existe una conexión interna no sólo entre valor y dinero, sino también entre dinero y capital, no podemos tener uno de ellos sin el otro. 
Probablemente para hacer la obra más popular, Marx omitió en "El Capital" esta transición categorial del dinero al capital. Pero esto tuvo como consecuencia que el lector podría pensar que el análisis de la mercancía y del dinero es independiente del análisis del capital, una apariencia que contribuiría entonces a hacer plausible la idea de que Marx analizaría en los tres primeros capítulos una "producción simple de mercancías" precapitalista. La separación de los tres primeros capítulos y la falta de una transición al capital (al nivel de categorías) también dieron alguna plausibilidad a la idea de un "socialismo de mercado", que depende de la posibilidad de un sistema de mercado global sin capital.

Evidentemente Marx no omitió la transición categorial del dinero al capital porque perdiera la confianza en la necesidad de esta transición - los elementos necesarios para esta transición todavía pueden encontrarse en el análisis de "El Capital". Cuando discute sobre el atesoramiento, Marx presenta claramente la contradicción entre el carácter ilimitado del dinero en sentido cualitativo y sus límites siempre vigentes en sentido cuantitativo (MEW 23, p. 147; p. 161). Esta contradicción encuentra su forma "racional" de movimiento sólo en la circulación de dinero como capital, por lo que Marx puede decir en el capítulo 4, "mientras que el atesorador es solamente el capitalista insensato, el capitalista es el atesorador racional" (während der Schatzbildner nur der verrückte Kapitalist, ist der Kapitalist der rationelle Schatzbildner, MEW 23, p. 168; p. 187). También otros argumentos utilizados en los "Grundrisse" y en el "Urtext" sobre la necesidad de la transición del dinero al capital pueden encontrarse en una lectura atenta de los capítulos 3 y 4 de "El Capital". Lo que falta es sólo que Marx reúna explícitamente estos argumentos.

\subsection{Teoría monetaria y no-monetaria del capital y crisis}

A pesar de que falte la transición categorial explícita del dinero al capital, el carácter monetario del capital aparece expresado también en "El Capital". En el capítulo 4 de "El Capital", cuando Marx está discutiendo el concepto general de capital, acentúa que el valor en proceso, que es capital, necesita el dinero como "forma autónoma" (selbständige Form), por la cual el valor "constata su identidad consigo mismo" (seine Identität mit sich selbst konstatiert, MEW 23, p. 169; p. 188). Aunque el capital no es ni dinero ni mercancía, sino el proceso de valorización, el dinero desempeña un papel muy especial en este proceso: sólo en la forma de dinero puede el capital basarse en sí mismo y puede medir el grado de valorización.

El carácter específicamente monetario del capital fue durante mucho tiempo ampliamente ignorado en la tradición marxista. En muchas discusiones marxistas sobre economía política se impuso el libro I de "El Capital". Puesto que la producción era la cuestión predominante de la mayor parte del libro I y puesto que este libro era tan influyente, la tradición marxista tuvo en algunos sentidos un sesgo 
hacia la producción. El dinero y el intercambio eran entendidos como "meros" fenómenos de la circulación. Las cosas reales, los puntos importantes parecían encontrarse en la esfera de la producción: la explotación, el desarrollo de las fuerzas productivas, la acumulación, etc.

Sin embargo, el dinero y los aspectos monetarios del capital tienen un papel crucial a lo largo de los tres libros de "El Capital". En el libro primero, como se mencionó anteriormente, se introdujo una noción monetaria de capital en el capítulo cuatro. En las siguientes secciones, en las que se analizan la producción de plusvalor absoluto y relativo, así como la acumulación, el dinero no tiene un papel crucial. Pero esto no significa que se pueda investigar el capital sin el dinero. Esta ausencia del dinero como una cuestión de la exposición es consecuencia sobre todo del tema particular que se investiga: el proceso de producción capitalista como tal sin su mediación por la circulación. Entonces el dinero aparece otra vez en algunos puntos decisivos del libro segundo, con respecto a la posibilidad de realizar el plusvalor social total, que es una cuestión crucial para el proceso de circulación del capital social global. Y sobre todo temas acerca del dinero en el libro III. En la sección sobre el interés y el crédito, los asuntos del dinero vuelven otra vez al centro de la consideración - pero esta sección está inacabada y es extremadamente fragmentaria, y durante mucho tiempo fue excluida generalmente del debate.

Desde comienzos de la década de 1990 el manuscrito original de Marx del libro tercero está disponible en MEGA II/4.2. Con ello se hizo evidente que Engels, para hacer que el manuscrito fuera más legible, realizó muchos cambios en su edición del libro tercero. Las intervenciones más extensas tuvieron lugar en la gran sección sobre el interés y el crédito. El material fue sustancialmente reordenado y en muchas partes reformulado, lo que oscureció parcialmente las intenciones de Marx. En el futuro, cualquier discusión científica de la teoría marxiana del crédito tendría que basarse en el manuscrito original y no en la edición de Engels.

También la recepción de la teoría marxiana de las crisis está profundamente influenciada por los cambios realizados por Engels. Las consideraciones más amplias acerca de la teoría de las crisis en "El Capital" las podemos encontrar después de la exposición de Marx de su famosa ley de la caída tendencial de la tasa de beneficio. En el manuscrito original de Marx es evidente que tenemos sólo notas que están muy lejos de una teoría coherente. Engels, en su intento de presentar las cosas al lector tan acabadas como fuera posible, reordenó y parcialmente acortó el material, insertó todos los títulos y subtítulos de tal modo que para el lector no sólo surgía la impresión de que la teoría de la crisis está prácticamente completa (incluso sin considerar el capital que devenga interés y las relaciones de crédito), y además la teoría de las crisis parece ser una consecuencia de la ley de la tasa de beneficio. Pero esto es evidentemente un efecto de edición de Engels. 
El modo de lectura que siguió la disposición de Engels encajaba muy bien con el punto de vista orientado a la producción, que fue sostenido tomando el libro primero de "El Capital" como un todo casi completo. Basando la teoría de las crisis tan fuertemente en la caída tendencial de la tasa de beneficio, se reduce la teoría de las crisis a algo que está enraizado sólo en el proceso de producción. El hecho de que la producción capitalista y la circulación capitalista forman una unidad es oscurecido por esa perspectiva, y además se reduce la teoría de las crisis en un aspecto muy grave.

Con respecto a la totalidad de las categorías presentadas por Marx, la teoría de las crisis no puede presentarse después de la ley del beneficio: la tasa media de beneficio no está aún plenamente desarrollada (esto sólo tiene lugar con el análisis del capital mercantil en la sección siguiente), y sobre todo faltan todavía el capital que devenga interés y el crédito. Sólo después de que se han realizado estos análisis, lo que significa sólo durante o después de la quinta sección del libro tercero de "El Capital", es posible un tratamiento adecuado de la teoría de las crisis. Algunos indicios de esto pueden encontrarse en el análisis inacabado de Marx sobre el sistema crediticio.

El tipo de recepción de la teoría de las crisis descrito anteriormente ha conducido, por un lado, a enconados intentos de defender a toda costa la ley de la caída tendencial de la tasa de beneficio, aunque esta ley puede ser rebatida con argumentos muy simples (para un tratamiento crítico de los diferentes intentos de defender esta ley, véase Heinrich 2006, capítulo 8.4); todavía está ampliamente extendida la idea de que sin esta ley no habría ningún fundamento para una teoría marxiana de las crisis. Por otro lado, la orientación unilateral hacia la producción fue durante mucho tiempo un obstáculo para concebir los procesos monetarios y financieros de una manera adecuada. A menudo una especie de crisis de sobreproducción (o sobreacumulación), enraizada solamente en las condiciones de producción, fue considerada como la única "crisis real", con el dinero y las finanzas como momentos no muy fundamentales.

Hoy en día, especialmente en algunas partes del debate sobre la globalización y la crisis financiera, la producción capitalista se confronta con los procesos financieros de tal manera que la producción suena como algo real, sano y bueno, y los procesos financieros suenan como algo virtual, enfermo y malo. Pero no hay dos mundos capitalistas diferentes, uno bueno y uno malo, uno real y uno virtual. Hay sólo un capitalismo, una unidad capitalista de producción y circulación.

De la misma manera que necesitamos una teoría monetaria del valor, también necesitamos una teoría monetaria del capital y de las crisis. No se trata de sustituir el sesgo orientado hacia la producción por un sesgo orientado a la circulación, sino de concebir la unidad capitalista de producción y circulación. 


\section{Observaciones finales}

En el análisis del capitalismo desde una perspectiva de la emancipación social, la crítica marxiana de la economía política es la herramienta más poderosa que tenemos. Pero al usar esta herramienta tenemos que ser conscientes de sus límites:

1) La crítica de Marx está incompleta, no pudo cumplir sus propios planes. Por lo tanto, tenemos que seguir adelante, no sólo con análisis empíricos, sino también con la investigación teórica, con la construcción y el desarrollo de las categorías.

2) La exposición de Marx muestra un cierto número de ambivalencias fundamentales que debemos examinar cuidadosamente, criticar y eliminar, con el fin de lograr un uso apropiado de su crítica de la economía política.

Criticar a Marx es exactamente lo que él, como un científico serio, esperaba de sus lectores. Al final del prefacio a "El Capital", escribió:

"Todo juicio de la crítica científica es bienvenido para mí" (MEW 23, p. 17; p. 9).

Y en una carta a Engels del 18 de julio de 1877 subrayó que "la falta de miramientos es la primera condición toda crítica" (Rücksichtslosigkeit - erste Bedingung aller Kritik, MEW 34, p. 48). Esto también debe aplicarse a nuestro tratamiento de Marx.

\section{Literatura}

Althusser, Louis (1965): Pour Marx, Paris: Maspero (La revolución teórica de Marx, México: Siglo XXI, 1968).

BACKHAUS, Hans Georg (1997): Dialektik der Wertform, Freiburg: Ça ira.

HeINRICH, Michael (1996/97): Engels' edition of the third volume of "Capital" and Marx's original manuscript, in: Science and Society, vol. 60, n. 4, pp. 195-210. HeINRICH, Michael (2004): Kritik der politischen Ökonomie. Eine Einführung, Stuttgart: Schmetterling (Crítica del economía política. Una introducción a "El Capital " de Marx, Madrid: Escolar y Mayo, 2008).

HeInRICH, Michael (2006): Die Wissenschaft vom Wert. Die Marxsche Kritik der politischen Ökonomie zwischen wissenschaftlicher Revolution und klassischer Tradition, 4th edition, Münster: Westfälisches Dampfboot.

HeInRICH, Michael (2008): Wie das Marxsche Kapital lesen?, Stuttgart: Schmetterling.

HeINRICH, Michael (2009): Reconstruction or Deconstruction? Methodological controversies about Value and Capital, and New Insights from the Critical Edition, in: Riccardo Bellofiore, Roberto Fineschi (eds.), Re-reading Marx. New Perspectives after the Critical Edition, Palgrave Macmillan, pp. 71-98. 
MARX, Karl (1859): Zur Kritik der politischen Ökonomie, MEW 13 (Contribución a la crítica de la economía política, México: Siglo XXI, 1980).

MARX, Karl (1867-94): Das Kapital. Kritik der politischen Ökonomie, 3 Bde., MEW 23-25 (El Capital. Crítica de la economía política (libros I, II y III), México: Siglo XXI, 1975).

MARX, Karl (1871/72): Ergänzungen und Veränderungen zum ersten Band des “Kapitals", MEGA II/6.

RuBIN, Isaac I. (1928): Essays in Marxian Value Theory, Moscow (English: Detroit, 1972; German: Studien zur Marxschen Werttheorie, Frankfurt/Main, 1973).

Michael Heinrich

Hochschule für Technik und Wirtschaft

Berlin (Germany)

m.heinrich@prokla.de 